ERRATUM

\title{
Association of thyroid function with estimated glomerular filtration rate in a population-based study: the HUNT study
}

Bjørn Olav Åsvold, Trine Bjøro and Lars J Vatten

The journal and the authors apologise for an error in this article published in the European Journal of Endocrinology (2011) $164101-105$. The caption to Table 4 on page 104 was wrongly printed and the correct caption and table is printed in full below.

European Journal of Endocrinology 164316

Table 4 Geometric mean eGFR $\left(\mathrm{ml} / \mathrm{min}\right.$ per $\left.1.73 \mathrm{~m}^{2}\right)$ by categories of thyroid function and age, adjusted for sex, age and smoking.

\begin{tabular}{|c|c|c|c|c|c|c|}
\hline & \multicolumn{3}{|c|}{ Age $<70$ years $(n=22071)$} & \multicolumn{3}{|c|}{ Age $\geq 70$ years $(n=7409)$} \\
\hline & eGFR & & & eGFR & & \\
\hline \multicolumn{7}{|c|}{ Hyperthyroidism } \\
\hline Overt & 120.5 & 113.5 & 128.0 & 87.0 & 78.9 & 96.0 \\
\hline Subclinical & 91.7 & 89.9 & 93.4 & 76.4 & 73.4 & 79.5 \\
\hline \multicolumn{7}{|l|}{ TSH (mU/l) } \\
\hline $0.50-1.4$ & 89.9 & 89.6 & 90.3 & 75.3 & 74.4 & 76.2 \\
\hline $1.5-2.4$ & 88.5 & 88.1 & 88.9 & 73.5 & 72.6 & 74.4 \\
\hline $2.5-3.5$ & 87.0 & 86.3 & 87.7 & 72.6 & 71.4 & 73.8 \\
\hline \multicolumn{7}{|l|}{ Hypothyroidism } \\
\hline Subclinical & 86.5 & 85.5 & 87.4 & 70.7 & 69.3 & 72.2 \\
\hline Overt & 83.3 & 80.5 & 86.4 & 68.3 & 63.3 & 73.6 \\
\hline
\end{tabular}

